\title{
Características epidemiológicas de la enfermedad periodontal e higiene oral en mujeres en etapa de embarazo y lactancia materna
}

Epidemiology of periodontal disease and oral hygiene in pregnant and breastfeeding women of a marginal Ivonne Yllesca-Yllesca ${ }^{1, a}$, Jorge Enrique Manrique-Chávez ${ }^{1,2, b}$, Beatriz Del Carmen Chávez-Reátegui 1,2,c.

\section{RESUMEN}

Objetivo: Comparar las características epidemiológicas de la enfermedad periodontal y la higiene oral entre mujeres que se encuentren en etapa de gestación y mujeres que se encuentran en periodo de lactancia de una comunidad urbano marginal de Lima-Perú en el 2014. Materiales y Métodos: Cuarenta mujeres agrupadas según el trimestre de gestación y cuarenta mujeres agrupadas según el bimestre de lactancia, fueron evaluadas empleando el índice de higiene oral simplificado, índice periodontal comunitario y el índice de pérdida de fijación. Resultados: Se realizó las pruebas estadísticas Chi Cuadrado y Test Exacto de Fisher para comparar ambos grupos. No se encontró diferencias estadísticamente significativas entre gestantes y lactantes al comparar la higiene oral, el sangrado al sondaje y la pérdida de fijación. Sin embargo se halló diferencias estadísticamente significativas con respecto a las bolsas periodontales, siendo más prevalentes en gestantes. Además es importante mencionar que las bolsas periodontales disminuyeron progresivamente a medida que avanzaban los trimestres de lactancia. Conclusión:Durante el embarazo, se presentaron más características epidemiológicas de enfermedad periodontal que en el periodo de lactancia materna, a pesar de que, ambos grupos tuvieron las mismas condiciones de higiene oral.

PALABRAS CLAVE: Enfermedades periodontales, higiene bucal, salud bucal, embarazo, lactancia materna. 


\section{SUMMARY}

Objective: To compare the epidemiology of periodontal disease and oral hygiene among women who are in gestation and women who are breast-feeding from a marginal urban community in Lima- Peru in 2014. Materials and Methods: Forty women grouped by trimester of gestation and forty women grouped by bimester of lactation were evaluated using the simplified oral hygiene index, community periodontal index and the loss of attachment index. Results: Statistical tests Chi Square and Fisher Exact Test was performed to compare both groups. No statistically significant differences between pregnant and lactating comparing oral hygiene, bleeding on probing and loss of attachment was found. However, significant differences were found with respect to the periodontal pockets, being most prevalent in pregnant women. It is also important to mention that periodontal pockets progressively decreased progressed during bimester of lactation. Conclusion:During pregnancy, more epidemiological characteristics of periodontal disease were presented than in the period of breastfeeding, even though both groups had the same conditions of oral hygiene.

\section{KEY WORDS: Periodontal diseases, oral hygiene, oral health, pregnancy, breast feeding.}

\section{INTRODUCCIÓN}

La inflamación gingival iniciada por placa y exacerbada por hormonas esteroideas sexuales endógenas se define como gingivitis del embarazo (1) y afecta a un $36 \%-100 \%$ de las mujeres embarazadas $(2,3)$. El aumento de dichas hormonas durante el embarazo influencia en los tejidos periodontales afectando el sistema inmune local, la vascularidad gingival, el biofilm y las células específicas del periodonto (4). Después del parto, la gingiva se recupera espontáneamente a condiciones similares a las que presentaba al principio del embarazo (5-8). Por el contrario, un estudio no pudo demostrar ninguna correlación entre el aumento de hormonas femeninas y el aumento de la inflamación gingival (9); y otra investigación informó que la placa es el principal implicado en el aumento de la inflamación gingival durante el embarazo y después del parto(10).

Por otra parte la enfermedad periodontal ha demostrado ser significativamente más severa en mujeres embarazadas en comparación con mujeres no embarazadas, a pesar que la higiene bucal de ambos grupos fue comparable (11). Un hallazgo similar ha sido reportado en un estudio donde se comparó embarazadas y mujeres en post-parto. (3)

Por otro lado muchos de los estudios previos llevados a cabo en mujeres embarazadas, no miden la pérdida de fijación durante el embarazo, lo que podría haber dado lugar a sub o sobre-estimación de los niveles de enfermedad.
El objetivo de este estudio fue comparar las características epidemiológicas de la enfermedad periodontal y la higiene oral entre mujeres que se encuentren en etapa de gestación y mujeres que se encuentran en periodo de lactancia de una comunidad urbano marginal de Lima-Perú en el 2014.

\section{MATERIAL Y MÉTODOS}

Es un diseño cuantitativo, tipo observacional, analítico, de corte transversal y prospectivo. Tuvo como población de estudió a mujeres embarazadas y lactantes de la comunidad "Catalina Huanca" del Agustino en el 2014, de las cuales se tomó una muestra de 40 embarazadas y 40 lactantes, según el cálculo muestral, luego de aplicar la fórmula estadística.

Los criterios de inclusión fueron: estar en periodo de embarazo o lactancia, residir en la comunidad "Catalina Huanca" del Agustino en el 2014, edad de 15 hasta 49 años, tener tarjeta de control pre-natal. Los criterios de exclusión fueron: haber recibido tratamiento periodontal previo, tener embarazo de alto riesgo o incapacidad para alimentar a su hijo con su propia leche materna y tener riesgo sistémico.

Antes de realizar la recopilación de información, el evaluador fue calibrado con un Gold Estándar (Especialista en Periodoncia). Posteriormente, se realizó la medición de Confiabilidad Interevaluador empleando el test de Kappa de Cohen sobre las mediciones de cada instrumento, obteniendo una puntuación de 0,94 . 
Se obtuvo el permiso del jefe del Puesto de Salud "Catalina Huanca" para acceder a la base de datos de mujeres embarazadas y lactantes y hacer el levantamiento epidemiológico. Al no tratarse de un estudio experimental, no fue necesario aplicar medidas de enmascaramiento de la población o muestra, por el contrario, el estudio se enfocó en disponer de una línea base epidemiológica de la población bajo evaluación.

Para seleccionar la muestra se procedióa aplicar un muestreo aleatorio simple sin reposición de elementos empleando una tabla de números aleatorios simples. Después, el investigador reunió a las mujeres seleccionadas en el puesto de salud y verificó, en sus historias clínicas, si cumplían con los criterios de inclusión del estudio. Posteriormente se procedió a realizar el examen periodontal, previa firma del consentimiento informado (o asentimiento en el caso de las menores de 18 años). En caso de no aceptación, se escogió a la siguiente participante en forma aleatoria simple hasta completar el tamaño de muestra requerida.

Después de concluir con el examen periodontal, el evaluador procedió a desechar todo el material no reutilizable siguiendo las normas de bioseguridad para desechos biológicos y realizar la desinfección química del material reutilizable. Este procedimiento se realizó con todas las mujeres que formaron parte de la muestra. Los datos demográficos y clínicos fueron recolectados en fichas epidemiológicas.

Para la medición de la higiene oral, se utilizó el índice de higiene oral simplificado y para determinar las características epidemiológicas de la enfermedad periodontal se empleó el índice periodontal comunitario del 2013 y el índice de pérdida de fijación (ambos de la Organización Mundial de la Salud - OMS), los cuales utilizan los parámetros clínicos: sangrado al sondaje, profundidad al sondaje y pérdida de fijación. Para evitar sesgos por la hora de alimentación, todas las mujeres fueron evaluadas por las mañanas, durante el horario de atención del establecimiento, luego de pasar revisión por el servicio de obstetricia (visita de control).

Una vez obtenidos los datos, se procedió a realizar el análisis estadístico, se realizó las pruebas Chi Cuadrado de Pearson y Test Exacto de Fisher para determinar la relación entre el estado periodontal en mujeres en etapa de embarazo y periodo de lactancia; estas pruebas también fueron usadas para determinar la relación entre la pérdida de fijación periodontal en mujeres en etapa de gestación y periodo de lactancia. Se tomó en cuenta un valor de $\mathrm{p}<0,05 \mathrm{y}$ el intervalo de confianza del 95\% como el nivel de significancia estadística. Para el análisis estadístico se empleó el software estadístico IBM SPSS Statistics v. 19.0 (SPSS Inc.).El protocolo de investigación fue revisado y aprobado por el Comité de Ética e Investigación de la Universidad Peruana Cayetano Heredia.

\section{RESULTADOS}

Se evaluaron ochenta mujeres, cuarenta embarazadas y cuarenta lactantes, todas mayores de 15 años de edad distribuidas en cuatro grupos etarios (Tabla 1), entre abril y junio del 2014. Las mujeres fueron agrupadas según el trimestre de embarazo o bimestre de lactancia en el que se encontraban al ser examinadas. Se observó que el $90 \%$ de embarazadas y el $95 \%$ de lactantes presentaron condiciones de higiene oral aceptables. Al comparar estos resultados, empleando las pruebas Chi cuadrado y Test exacto de Fisher, no se halló diferencia estadísticamente significativa ( $p$ $>0,05$ ) (Tabla 2). El 100\% de embarazadas y el 100\% de lactantes presentaron sangrado al sondaje; por ende no fue necesario aplicar ninguna prueba estadística (Tabla 3). Por otro lado,el 90\% de embarazadas y el 33\% de lactantes presentaron bolsas periodontales.Al realizar el análisis estadístico, empleando Chi cuadrado y Test exacto de Fisher, se obtuvo una diferencia estadísticamente significativa $(\mathrm{p}<0.05)$, lo que indica que en el periodo de embarazo hubieron más mujeres con bolsas periodontales que en el periodo de lactancia (Tabla 4). Cabe resaltar que las bolsas periodontales aumentaron progresivamente a medida que avanzaban los trimestres de embarazo, alcanzando un máximo valor en el tercer trimestre, después del parto las bolsas periodontales disminuyeron en el primer bimestre de lactancia hasta mantenerse constantes en el segundo y tercer bimestre (Gráfico 1). Con respecto a la pérdida de fijación, no se presentó esta condición patológica en mujeres lactantes (Tabla 5), mientras que el $10 \%$ de embarazadas presentó pérdida de fijación mayor a $4 \mathrm{~mm}$ (Tabla 6). Al comparar dichos resultados, empleando las pruebas Chi cuadrado y Test exacto de Fisher, no se halló diferencia estadísticamente significativa $(\mathrm{p}>0,05)$. 
Tabla 1. Distribución según grupo etario de mujeres embarazadas y en periodo de lactancia. Puesto de Salud “Catalina Huanca”, Distrito El Agustino. Lima- Perú, 2014.

\begin{tabular}{ccccc}
\hline \multirow{2}{*}{ Rango de edades } & \multicolumn{2}{c}{ Embarazadas } & \multicolumn{2}{c}{ Lactantes } \\
& $\mathbf{N}^{\mathbf{o}}$ & $\mathbf{\%}$ & $\mathbf{N}^{\mathbf{o}}$ & $\mathbf{\%}$ \\
\hline De 15 a 21 & 18 & $45 \%$ & 17 & $43 \%$ \\
De 22 a 28 & 11 & $28 \%$ & 9 & $23 \%$ \\
De 29 a 35 & 7 & $18 \%$ & 9 & $23 \%$ \\
De 36 a 42 & 4 & $10 \%$ & 5 & $13 \%$ \\
Total & 40 & $100 \%$ & 40 & $100 \%$ \\
\hline
\end{tabular}

Tabla 2. Condición de higiene oral en mujeres embarazadas y en periodo de lactancia. Puesto de Salud “Catalina Huanca”, Distrito El Agustino. Lima- Perú, 2014.

\begin{tabular}{ccccccccc}
\hline \multirow{2}{*}{ Estado } & \multicolumn{2}{c}{$\begin{array}{c}\text { Adecuada } \\
\mathbf{0}-\mathbf{1 . 2}\end{array}$} & \multicolumn{2}{c}{ Aceptable } & \multicolumn{2}{c}{ Deficiente } & \multicolumn{2}{c}{ Total } \\
& $\mathbf{N}^{\mathbf{0}}$ & $\mathbf{1 . 3}-\mathbf{3}$ & $\mathbf{N}^{\mathbf{0}}$ & $\mathbf{\%}$ & $\mathbf{N}^{\mathbf{0}}$ & $\mathbf{3 . 1}-\mathbf{6}$ & $\mathbf{N}^{\mathbf{0}}$ & $\mathbf{\%}$ \\
\hline Embarazo & 0 & $0 \%$ & 36 & $45 \%$ & 4 & $5 \%$ & 40 & $50 \%$ \\
Lactancia & 1 & $1,3 \%$ & 38 & $47,5 \%$ & 1 & $1,3 \%$ & 40 & $50 \%$ \\
Total & 1 & $1,3 \%$ & 74 & $92,5 \%$ & 5 & $6,3 \%$ & 80 & $100 \%$ \\
\hline
\end{tabular}

$(\mathrm{p}>0,05)$

Tabla 3. Sangrado al sondaje en mujeres embarazadas y en periodo de lactancia. Puesto de Salud “Catalina Huanca”, Distrito El Agustino. Lima- Perú, 2014.

\begin{tabular}{clc}
\hline Estado & \multicolumn{2}{c}{ Sangrado al Sondaje } \\
& $\mathbf{N}^{\mathbf{0}}$ & \% \\
\hline Embarazo & 40 & 50 \\
Lactancia & 40 & 50 \\
Total & 80 & 100 \\
\hline
\end{tabular}

Tabla 4. Bolsas periodontales en mujeres embarazadas y en periodo de lactancia. Puesto de Salud “Catalina Huanca”, Distrito El Agustino. Lima- Perú, 2014.

\begin{tabular}{cccccc}
\hline & \multicolumn{4}{c}{ Bolsas periodontales } \\
Estado & $\mathbf{N}^{\mathbf{0}}$ & $\mathbf{\%}$ & $\mathbf{N}^{\mathbf{0}}$ & $\mathbf{\%}$ & Total \\
\hline Embarazo & 4 & 5 & 36 & 45 & $\mathbf{N}^{\mathbf{0}}$ \\
Lactancia & 27 & 34 & 13 & 16 & 40 \\
Total & 31 & 39 & 49 & 61 & 40 \\
\hline
\end{tabular}

$(\mathrm{p}<0,05)$ 


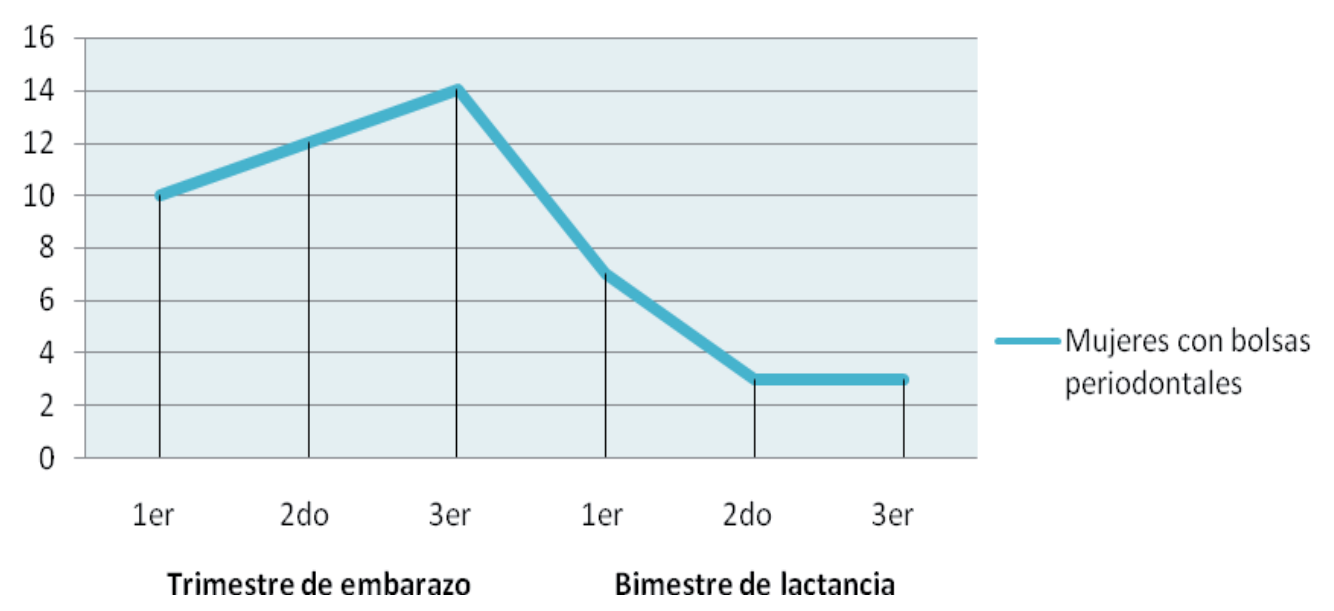

Gráfico 1. Número de bolsas periodontales en mujeres gestantes y en periodo de lactancia. Puesto de Salud “Catalina Huanca”, Distrito El Agustino. Lima, Perú, 2014.

Tabla 5. Pérdida de fijación en mujeres gestantes y en periodo de lactancia.Puesto de Salud “Catalina Huanca”, Distrito El Agustino. Lima- Perú, 2014.

\begin{tabular}{|c|c|c|c|c|c|c|c|c|c|c|c|c|}
\hline \multirow{3}{*}{ Estado } & \multicolumn{8}{|c|}{ Pérdida de fijación periodontal } & & & & \\
\hline & \multicolumn{2}{|c|}{$0-3 \mathrm{~mm}$} & \multicolumn{2}{|c|}{$4-5 \mathrm{~mm}$} & \multicolumn{2}{|c|}{$6-8 \mathrm{~mm}$} & \multicolumn{2}{|c|}{$\begin{array}{c}9-11 \\
\mathrm{~mm}\end{array}$} & \multicolumn{2}{|c|}{$\begin{array}{l}12 \mathrm{~mm} \text { a } \\
\text { más }\end{array}$} & \multicolumn{2}{|c|}{ Total } \\
\hline & $\mathrm{n}$ & $\%$ & $\mathrm{n}$ & $\%$ & $\mathrm{n}$ & $\%$ & $\mathrm{n}$ & $\%$ & $\mathrm{n}$ & $\%$ & $\mathrm{n}$ & $\%$ \\
\hline Embarazo & 36 & 90 & 3 & 7,5 & 1 & 2,5 & 0 & 0 & 0 & 0 & 40 & 50 \\
\hline Lactancia & 40 & 100 & 0 & 0 & 0 & 0 & 0 & 0 & 0 & 0 & 40 & 50 \\
\hline Total & 76 & 90 & 3 & 7.5 & 1 & 2.5 & 0 & 0 & 0 & 0 & 80 & 100 \\
\hline
\end{tabular}

$(p>0,05)$

Tabla 6. Pérdida de fijación en mujeres gestantes según trimestre de embarazo. Puesto de Salud “Catalina Huanca”, Distrito El Agustino. Lima- Perú, 2014.

\begin{tabular}{|c|c|c|c|c|c|c|c|c|c|c|c|c|}
\hline \multirow{3}{*}{ Estado } & \multicolumn{8}{|c|}{ Pérdida de fijación periodontal } & & & & \\
\hline & \multicolumn{2}{|c|}{$0-3 \mathrm{~mm}$} & \multicolumn{2}{|c|}{$4-5 \mathrm{~mm}$} & \multicolumn{2}{|c|}{$6-8 \mathrm{~mm}$} & \multicolumn{2}{|c|}{$9-11 \mathrm{~mm}$} & \multicolumn{2}{|c|}{12 mm a más } & \multicolumn{2}{|c|}{ Total } \\
\hline & $\mathrm{n}$ & $\%$ & $\mathrm{n}$ & $\%$ & $\mathrm{n}$ & $\%$ & $\mathrm{n}$ & $\%$ & $\mathrm{n}$ & $\%$ & $\mathrm{n}$ & $\%$ \\
\hline 1er Trimestre de embarazo & 13 & 32,5 & 0 & 0 & 0 & 0 & 0 & 0 & 0 & 0 & 13 & 32,5 \\
\hline 2do Trimestre de embarazo & 12 & 30,0 & 1 & 2,5 & 0 & 0 & 0 & 0 & 0 & 0 & 13 & 32,5 \\
\hline 3er Trimestre de embarazo & 11 & 27,5 & 2 & 5 & 1 & 2,5 & 0 & 0 & 0 & 0 & 14 & 35,0 \\
\hline Total & 36 & 90 & 3 & 7,5 & 1 & 2,5 & 0 & 0 & 0 & 0 & 40 & 100 \\
\hline
\end{tabular}

$(\mathrm{p}>0,05)$ 


\section{DISCUSIÓN}

En el presente estudio se observó que embarazadas y lactantes mostraron los mismos niveles de placa. Resultados similares a los nuestros fueron reportados por Figuero y colaboradores en su revisión sistemática que tuvo como objetivo obtener una estimación cuantitativa de la asociación entre el embarazo y la inflamación gingival, para lo cual analizaron 33 estudios longitudinales y transversales, de los cuales 14 se emplearon para determinar los niveles de placa dental, dichos autores no encontraron diferencia estadísticamente significativa entre los índices de placa bacteriana en embarazadas y mujeres en post-parto (12). Por otro lado es bastante conocido el rol y la estrecha relación de la placa bacteriana en el inicio y progresión de la enfermedad periodontal, debido a esto, Tilakaratne y colaboradores afirmaron que para el correcto estudio del rol del embarazo,en la progresión de la enfermedad periodontal, es necesario aislar los efectos de la placa sobre el periodonto, por lo que sugieren que la muestra estudiada presente niveles similares de placa bacteriana oral.

Por otro lado, se encontró un 100\% de prevalencia de sangrado al sondaje tanto en embarazadas como en lactantes, el sangrado al sondaje representa la mejor medida clínica de inflamación gingival (13). Nuestros resultados están de acuerdo con los de Loe y Silness, quienes evaluaron la incidencia y variación de la enfermedad periodontal en el embarazo y después del parto, examinando 121 embarazadas y 61 mujeres en post-parto, encontrando que el $100 \%$ de mujeres examinadas durante el embarazo y durante el post-parto mostraron signos de inflamación gingival (3).También existen investigaciones realizadas en población peruana, que muestran que la prevalencia de gingivitis en mujeres embarazadas y en post-parto es de 100\% (14-17). Es importante tener en cuenta que otros autores han reportado distintos porcentajes de prevalencia de gingivitis en embarazadas que varían ampliamente $(2,3)$, esto podría deberse a que los investigadores llevaron a cabo sus estudios en diferentes poblaciones, por ende, las muestras presentaron distintos orígenes étnicos, niveles socioeconómicos, y hábitos de cuidado oral.

Por otra parte, al evaluar la presencia de bolsas periodontales que fue definida como un sitio con profundidad al sondaje de $\geq 4 \mathrm{~mm}$, encontramos que en el periodo de embarazo hubo más mujeres con bolsas periodontales que en el periodo de lactancia. Resultados similares fueron obtenidos por Gursoy y colaboradores quienes compararon el estado periodontal de 30 mujeres a lo largo de su embarazo y hasta después de su periodo de lactancia con un grupo control de 24 mujeres no embarazadas, encontrando que el número de bolsas periodontales en el embarazo fue significativamente mayor que en el post parto y en el grupo control (8). Por su parte Loe y Silness manifiestan que el hecho que la profundidad de la bolsa se haya reducido después del parto parece indicar que la formación de la bolsa periodontal pudo ser causada por la inflamación de la encía libre (3).

Con respecto a la pérdida de fijación, ésta se mantuvo estable durante el embarazo y el periodo de lactancia, y no se encontró diferencia estadísticamente significativa $(p>0.05)$ entre ambos grupos. Nuestros hallazgos parecen estar de acuerdo con Miyazaki quien menciona que la formación de bolsas periodontales durante el embarazo se produce principalmente por la inflamación gingival en lugar de la destrucción del tejido periodontal; debido a que las bolsas periodontales disminuyen significativamente después del parto y los niveles de pérdida de fijación no presentan diferencias significativas entre embarazadas y lactantes (18). Por otro lado, Tilakaratne y colaboradores en su investigación sobre el efecto del embarazo en el periodonto en una población rural de Sri-Lankan, examinaron 47 mujeres durante su embarazo y 3 meses post parto, además de un grupo control de 47 no embarazadas, los resultados de dicha investigación muestran que no hubo diferencia significativa entre las embarazadas y el grupo control (7). Los autores refieren que los niveles elevados de hormonas gestacionales durante un período de nueve meses no fueron suficientes para causar destrucción periodontal significativa, a pesar de sus efectos reportados en la barrera epitelial, en la vascularidad y en la matriz de tejido conectivo (7).

En conclusión durante el embarazo se presentaron más características epidemiológicas de enfermedad periodontal que en el periodo de lactancia materna, a pesar de que, ambos grupos tuvieron las mismas condiciones de higiene oral. 


\section{Correspondencia:}

Ivonne Yllesca-Yllesca

Correo electrónico: ivonne.yllesca@upch.pe

\section{REFERENCIAS BIBLIOGRÁFICAS}

1. Mariotti A. Sex steroid hormones and cell dynamics in the periodontium. Crit Rev Oral Biol Med.1994; 5(1):27-53.

2. Maier A, Orban B. Gingivitis in pregnancy. Oral Surg Oral Med Oral Pathol. 1949; 2(1):334-73.

3. Loe H, Silness, J. Periodontal disease in pregnancy. I. Prevalence and severity. Acta Odontol Scand. 1963; 21(1):533-51.

4. Guzmán Y. Severidad de la gingivitis asociada al embarazo en adolescentes. Tesis Bachiller. Lima, Perú: Universidad Mayor de San Marcos; 2005.

5. Löe H, Silness J. Periodontal disease in pregnancy. II. Correlation between oral hygiene and periodontal condition. Acta Odontol Scand. 1964; 22(1):121-35.

6. Cohen DW, Shapiro J, Friedman L, Kyle GC, Franklin S. A longitudinal investigation of the periodontal changes during pregnancy and fifteen months post-partum. II. J Periodontol. 1971; 42(1):653-7.

7. Tilakaratne A, Soory M, Ranasinghe A, Corea S, Ekanayake S, De Silva M. Periodontal disease status during pregnancy and 3 months post-partum, in a rural population of Sri-Lankan women. J Clin Periodontol. 2000; 27(1):787-92.

8. Gürsoy M, Pajukanta R, Sorsa T, Könönen E. Clinical changes in periodontium during pregnancy and postpartum. J Clin Periodontol. 2008; 35(1):576-83.

9. Jonsson R, Howland BE, Bowden GHW. Relationships between periodontal health, salivary steroids, and Bacteroides intermedius in males, pregnant and nonpregnant women. J Dent Res. 1988; 67(1):1062-9.

10. Carrillo-De-Albornoz A, Figuero E, Herrera D, Cuesta P, Bascones-Martinez A. Gingival changes during pregnancy: III. Impact of clinical, microbiological, immunological and sociodemographic factors on gingival inflammation. J Clin Periodontol. 2012; 39 (1):272-83.

11. Arafat AH. Periodontal status during pregnancy. J Periodontol. 1974; 45(1): 641-3.

12. Figuero E, Carrillo-de-Albornoz A, Martín C, Tobías A, Herrera D. Effect of pregnancy on gingival inflammation in systemically healthy women: a systematic review. J Clin Periodontol. 2013; 40 (1):457-73.

13. Newbrun E. Indices to measure gingival bleeding. J Periodontol. 1996; 67(1): 555-61.

14. Tremolada J. Prevalencia y severidad de gingivitis del embarazo y su correlación con las concentraciones de estradiol y progesterona. Tesis Bachiller. Lima, Perú: Universidad Nacional Mayor de San Marcos; 1984.

15. Santiago L. Gingivitis en gestantes y puérperas de altura y del nivel del mar. Acta Andina. 1994; 3(2):137-43.

16. Picasso M. Correlación entre estado nutricional, edad, higiene y experiencia de caries con gingivitis en embarazadas del distrito de San Juan de Lurigancho. Tesis Bachiller. Lima, Perú: Universidad Peruana Cayetano Heredia; 1995.

17. Velarde C. Estudio comparativo de la gingivitis a nivel del mar y a la altura. Tesis Bachiller. Lima, Perú: Universidad Peruana Cayetano Heredia; 1986.

18. Miyazaki H, Yamashita Y, Shirahama R, et al. Periodontal condition of pregnant women assessed by CPITN. J Clin Periodontol. 1991; 18 (1):751-4.

Recibido : 13/08/2015

Aceptado: 04/10/2015 